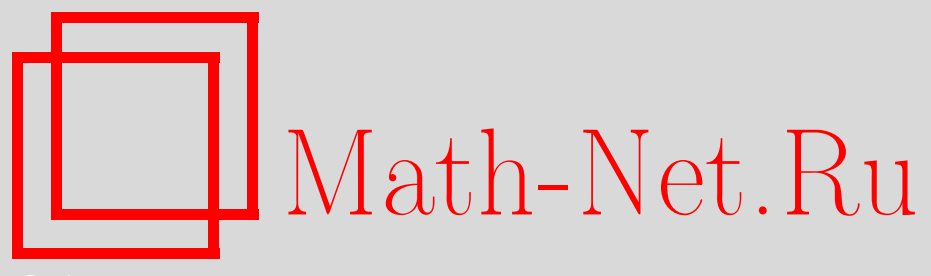

В. М. Бухштабер, Общий род Кричевера, УМН, 2010, том 65, выпуск 5, 187-188

DOI: https://doi.org/10.4213/rm9380

Использование Общероссийского математического портала Math-Net.Ru подразумевает, что вы прочитали и согласны с пользовательским соглашением http: //www . mathnet.ru/rus/agreement

Параметры загрузки:

IP: 35.174 .16 .151

26 апреля 2023 г., $11: 43: 43$

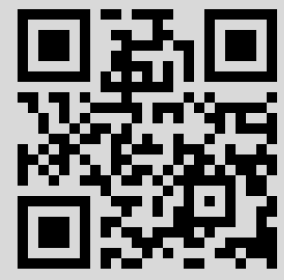




\section{Общий род Кричевера}

\section{В. М. Бухштабер}

В работе на основе общей модели Вейерштрасса эллиптической кривой

$$
V=\left\{(x: y: z) \in \mathbb{C} P^{2}: y^{2} z+\mu_{1} x y z+\mu_{3} y z^{2}=x^{3}+\mu_{2} x^{2} z+\mu_{4} x z^{2}+\mu_{6} z^{3}\right\}
$$

вводится общий эллиптический род Хирцебруха и общий род Кричевера.

Рассмотрим функцию

$$
\xi(u)=\xi(u ; v, w)=-\frac{1}{2} \frac{\wp^{\prime}(u)+\wp^{\prime}(w)}{\wp(u)-\wp(v)},
$$

где $\wp(u)=\wp\left(u ; g_{2}, g_{3}\right)$ - стандартная $\wp$-функция Вейерштрасса. Функция $(\xi(u)-1 / u)$ является регулярной в окрестности $u=0$. Введем функцию $\Phi(u)=\Phi(u ; v, w)=$ $1 / u+$ (регулярная функция) как решение уравнения $\Phi^{\prime}(u)+\xi(u) \Phi(u)=0$.

ЛЕмма 1. Функиия $\Phi(и)$ удовлетворяет уравнению

$$
\Phi^{\prime \prime}(u)-(2 \wp(u)-U(u)) \Phi(u)=\wp(v) \Phi(u),
$$

где $U(u)=U(u ; v, w)=\xi(v ; u, w) \xi(v ; u,-w)$.

При $v=w$ уравнение (3) переходит в классическое уравнение Ламе и его решением является функция Бейкера-Ахиезера

$$
\Phi(u ; v, v)=\frac{\sigma(v-u)}{\sigma(v) \sigma(u)} \exp (\zeta(v) u) .
$$

Пусть $A=\sum A_{-2 k}$ - градуированное коммутативное ассоциативное кольцо без кручения и $f(u)=u+\sum_{k \geqslant 1} f_{k} u^{k+1}$, где $f_{k} \in A_{-2 k} \otimes \mathbb{Q}$. Ряд $\prod_{i=1}^{N} \frac{u_{i}}{f\left(u_{i}\right)}$ можно переписать в виде $L_{f}\left(\sigma_{1}, \ldots, \sigma_{k}, \ldots\right)$, где $\sigma_{k}-k$-я элементарная симметрическая функция от $u_{1}, \ldots, u_{N}$. Род Хирцебруха $L_{f}$ стабильно комплексного многообразия $M^{2 n}$ с касательными классами Чженя $c_{i}=c_{i}\left(\tau\left(M^{2 n}\right)\right)$ и фундаментальным циклом $\left\langle M^{2 n}\right\rangle$ задается формулой

$$
L_{f}\left(M^{2 n}\right)=\left(L_{f}\left(c_{1}, \ldots, c_{n}\right),\left\langle M^{2 n}\right\rangle\right) \in A_{-2 n} \otimes \mathbb{Q} .
$$

ОПредЕление 2. Род $L_{f}$ называется $A$-иелочисленным, если $L_{f}\left(M^{2 n}\right) \in A$ для любого стабильно комплексного многообразия $M^{2 n}$.

Пусть $F\left(t_{1}, t_{2}\right)$ - некоторая формальная группа над $A$. Тогда однозначно определен ряд $f(u) \in A \otimes \mathbb{Q}[[u]]$ такой, что $F\left(t_{1}, t_{2}\right)=f\left(g\left(t_{1}\right)+g\left(t_{2}\right)\right)$, где $f(g(t))=t$. Ряд $f(u)$ называется экспонентой группы $F\left(t_{1}, t_{2}\right)$, а ряд $g(t)$ - ее логарифмом.

Над кольцом кобордизмов $\Omega_{U}=\mathbb{Z}\left[a_{1}, \ldots, a_{n}, \ldots\right], \operatorname{deg} a_{n}=-2 n$, определена формальная группа $\mathscr{F}\left(t_{1}, t_{2}\right)=t_{1}+t_{2}+\sum a_{i j} t_{1}^{i} t_{2}^{j}, \operatorname{deg} a_{i j}=-2(i+j-1)$, геометрических кобордизмов [1], которая является универсальной формальной группой [2].

Каждый род Хирцебруха $L_{f}$ задает кольцевой гомоморфизм $L_{f}: \Omega_{U} \rightarrow A \otimes \mathbb{Q}$. Отображение, сопоставляющее формальной группе $F\left(t_{1}, t_{2}\right)=t_{1}+t_{2}+\sum \alpha_{i j} t_{1}^{i} t_{2}^{j}$, $\operatorname{deg} \alpha_{i j}=-2(i+j-1)$, над $A$ род Хирцебруха $L_{f}$, где $f(u)-$ экспонента этой группы, устанавливает взаимно однозначное соответствие между $A$-целочисленными родами и формальными группами $F\left(t_{1}, t_{2}\right)$ над $A$ такое, что $F\left(t_{1}, t_{2}\right)=t_{1}+t_{2}+\sum L_{f}\left(a_{i j}\right) t_{1}^{i} t_{2}^{j}$ (cм. [3]-[5]). 
Для кривой (1) положим $\operatorname{deg} x=-4, \operatorname{deg} y=-6, \operatorname{deg} z=0$. Тогда условие однородности дает $\operatorname{deg} \mu_{k}=-2 k, k=1,2,3,4,6$.

Пусть $U_{y}=\left\{(x: y: z) \in \mathbb{C} P^{2}, y \neq 0\right\}$. Образом кривой (1) при координатном отображении $U_{y} \rightarrow \mathbb{C}^{2}:(x: y: z) \rightarrow(t=-x / y, s=-z / y)$, является плоская кривая

$$
V=\left\{(t, s) \in \mathbb{C}^{2}: s=t^{3}+\mu_{1} t s+\mu_{2} t^{2} s+\mu_{3} s^{2}+\mu_{4} t s^{2}+\mu_{6} s^{3}\right\} .
$$

Из уравнения (5) следует, что $s=s(t) \in A[[t]]$. Здесь и далее $A=\mathbb{Z}\left[\mu_{1}, \ldots, \mu_{6}\right]$. В координатах $(t, s(t))$ групповая структура на эллиптической кривой (5) задает (см. [6]) формальную группу $F\left(t_{1}, t_{2}\right) \in A\left[\left[t_{1}, t_{2}\right]\right]$, которую мы будем называть общей эллиптической формалъной группой $F_{\mathrm{El}}\left(t_{1}, t_{2}\right)$.

ОПределЕниЕ 3 . Род Хирцебруха $L_{\mathrm{El}}$, задаваемый экспонентой $f_{\mathrm{El}}(u)$ формальной группы $F_{\mathrm{El}}\left(t_{1}, t_{2}\right)$, будем называть общим эллиптическим родом Хириебруха.

И. М. Кричевер [7] ввел род Хирцебруха, обладающий фундаментальным свойством жесткости на унитарных $S U$-многообразиях. В доказательстве результатов работы [7] важную роль играют методы теории абелевых функций и идеи теории интегрируемых систем. Свойства формальной группы, соответствующей роду Кричевера, описаны в [8].

ОПредЕЛЕНИЕ 4 . Общим родом Кричевера $L_{\mathrm{Kr}}$ будем называть род Хирцебруха $L_{f_{\mathrm{Kr}}}$, где $f_{\mathrm{Kr}}(u) \cdot \Phi(u ; v, w)=\exp \left(\mu_{1} u / 2\right)$.

Из формулы (3) следует, что род, введенный в [7], отвечает случаю $v=w$.

Группа $F_{\mathrm{El}}\left(t_{1}, t_{2}\right)$ и соответствующий ей род Хирцебруха детально изучены в [9]. Следующий результат получен совместно с Е. Ю. Буньковой.

Положим $b_{2}=\mu_{1}^{2}+4 \mu_{2}, b_{4}=\mu_{1} \mu_{3}+2 \mu_{4}, b_{6}=\mu_{3}^{2}+4 \mu_{6}$.

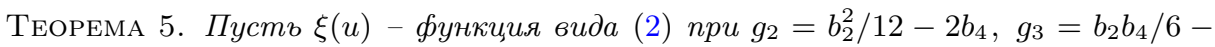
$b_{2}^{3} /(18 \cdot 12)-b_{6}, \wp(v)=b_{2} / 12, \wp^{\prime}(w)=-\mu_{3}$. Тогда:

1) $f_{\mathrm{El}}(u)\left(\mu_{1} / 2+\xi(u)\right)=1$, m.e. $\left(\ln f_{\mathrm{Kr}}(u)\right)^{\prime}=1 / f_{\mathrm{El}}(u)$;

2) для $\alpha=\wp^{\prime}(w) / \wp^{\prime}(v)=-\mu_{3} / \sqrt{\mu_{3}^{2}+4 \mu_{6}}$ имеет место формула

$$
f_{\mathrm{Kr}}(u)=\frac{\sigma(u) \sigma(v)}{\sigma(v+u)^{(1-\alpha) / 2} \sigma(v-u)^{(1+\alpha) / 2}} \exp \left(\frac{\mu_{1}}{2}-\alpha \zeta(v)\right) u ;
$$

3) $f_{\mathrm{Kr}}(u) \in A \otimes \mathbb{Q}[[u]]$.

Отметим, что общий род Кричевера не является $A$-целочисленным.

Пример 6. В случае $\mu_{1}=\mu_{3}=\mu_{4}=\mu_{6}=0$ род $L_{\mathrm{El}}\left(M^{2 n}\right)$ равен сигнатуре многообразия. Ему соответствует формальная группа $F_{\mathrm{El}}\left(t_{1}, t_{2}\right)=\left(t_{1}+t_{2}\right) /\left(1+\mu_{2} t_{1} t_{2}\right)$ с экспонентой $f_{\mathrm{El}}(u)=\mu_{2}^{-1 / 2} \tanh \sqrt{\mu_{2}} u$, и род $L_{\mathrm{Kr}}$ задается рядом $\mu_{2}^{-1 / 2} \sinh \sqrt{\mu_{2}} u$, который является экспонентой группы $F\left(t_{1}, t_{2}\right)=t_{1} \sqrt{1+\mu_{2} t_{2}^{2}}+t_{2} \sqrt{1+\mu_{2} t_{1}^{2}}$.

\section{Список литературы}

[1] С. П. Новиков, Изв. АН СССР. Сер. матем., 31:4 (1967), 855-951. [2] D. Quillen, Bull. Amer. Math. Soc., 75 (1969), 1293-1298. [3] С. П. Новиков, Изв. АН СССР. Сер. матем., 32:6 (1968), 1245-1263. [4] В. М. Бухштабер, Матем. сб., 83:4 (1970), 575-595. [5] В. М. Бухштабер, С. П. Новиков, Матем. сб., 84:1 (1971), 81-118. [6] J. Т. Tate, Invent. Math., 23 (1974), 179-206. [7] И. М. Кричевер, Матем. заметки, 47:2 (1990), 34-45. [8] В. М. Бухштабер, УMH, 45:3 (1990), 185-186. [9] V. M. Buchstaber, E. Yu. Bunkova, Elliptic formal group laws, integral Hirzebruch genera and Krichever genera, arXiv: 1010.0944.

B. M. Бухштабер (V. M. Buchstaber)

Математический институт им. В. А. Стеклова РАН

E-mail: buchstab@mi.ras.ru
Представлено С. П. Новиковым Принято редколлегией 30.08 .2010 